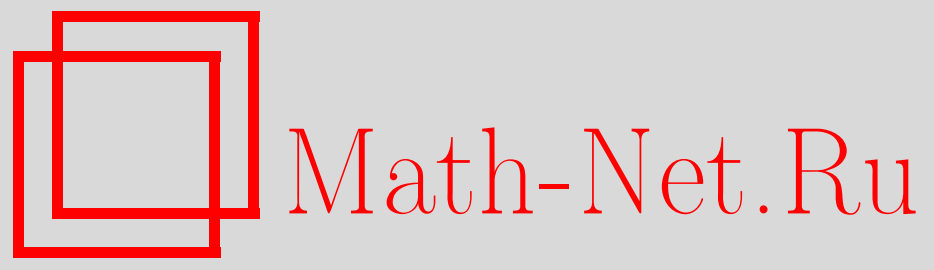

Л. В. Розовский, О сверхбольших уклонениях суммы независимых случайных величин с общим абсолютно непрерывным распределением, удовлетворяющим условию Крамера, Теория вероятн. и ее примен., 2003, том 48, выпуск 1, 78-103

DOI: https://doi.org/10.4213/tvp302

Использование Общероссийского математического портала MathNet.Ru подразумевает, что вы прочитали и согласны с пользовательским соглашением

http://www.mathnet.ru/rus/agreement

Параметры загрузки:

IP : 3.89 .185 .249

26 апреля 2023 г., 15:40:12

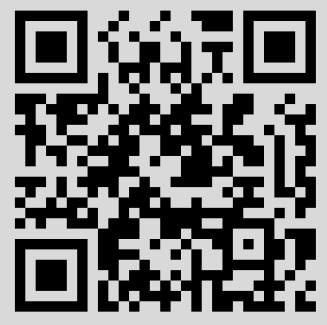


(c) $2002 \Gamma$.

РОЗОВСКИЙ Л. В.*

\section{О СВЕРХБОЛЬШИХ УКЛОНЕНИЯХ СУММЫ НЕЗАВИСИМЫХ СЛУЧАЙНЫХ ВЕЛИЧИН С ОБЩИМ АБСОЛЮТНО НЕПРЕРЫВНЫМ РАСПРЕДЕЛЕНИЕМ, УДОВЛЕТВОРЯЮЩИМ УСЛОВИЮ КРАМЕРА ${ }^{1)}$}

В заметке изучается асимптотическое поведение плотности суммы независимых одинаково распределенных случайных величин, имеющих абсолютно непрерывное распределение, которое удовлетворяет правостороннему условию Крамера.

Доказано, что для определенных классов таких распределений хорошо известные асимптотические представления в локальной и интегральной предельных теоремах остаются справедливыми в случае больших уклонений произвольно высокого порядка.

Ключевые слова и фразы: независимые случайные величины, плотность распределения, большие уклонения, условие Крамера.

1. Введение. Рассмотрим последовательность $\left\{X_{i}\right\}_{i \geqslant 1}$ независимых случайных величин с общим невырожденным распределением, удовлетворяющим правостороннему условию Крамера

$$
0<\lambda=\sup \left\{h: L(h)=\mathbf{E} e^{h X_{1}}<\infty\right\} \leqslant \infty .
$$

В дальнейшем будем также предполагать, что распределение $X_{1}$ является абсолютно непрерывным с плотностью $p(x)$.

Положим

$$
m(h)=(\ln L(h))^{\prime}, \quad \sigma^{2}(h)=m^{\prime}(h), \quad 0<h<\lambda .
$$

Из результатов [1] следует, что если при некотором целом $m \geqslant 1$

$$
\sup _{x} p^{* m}(x)<\infty
$$

(здесь и далее $p^{* m}$ обозначает $m$-кратную свертку плотности $p$ ), то

$$
p^{* n}(x)=\frac{1}{\sigma(h) \sqrt{2 \pi n}} L^{n}(h) e^{-h x}\left(1+\sum_{j=1}^{s-1} a_{j}(h) n^{-j}+O\left(n^{-s}\right)\right)
$$

${ }^{*}$ С.-Петербургская химико-фармацевтическая академия, ул. проф. Попова, 14, 197376 C.-Петербург, Россия; e-mail: math@spcpa.ru; L_Rozovsky@mail.ru

1) Работа выполнена при поддержке гранта РФФИ № 99-01-00724 и гранта ведуших научных школ № 00-15-96019. 
при $n \rightarrow \infty$, равномерно по $x, n m(\varepsilon) \leqslant x \leqslant n m\left(\lambda_{0}\right)$, где $h=h(x / n)-$ решение уравнения

$$
m(h)=\frac{x}{n},
$$

$\varepsilon, \lambda_{0}$ и целое $s$ - любые постоянные, удовлетворяющие условиям $0<\varepsilon<$

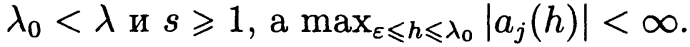

Заметим (см. [2], [3]), что $m(\varepsilon) \downarrow \mathbf{E} X_{1}$ при $\varepsilon \downarrow 0$, т.е. в приведенном выше утверждении вместо $m(\varepsilon)$ можно написать любое $\mu>\mathbf{E} X_{1}$.

Попытка расширить зону выполнения равенства (1.4) путем устремления $\lambda_{0}$ к $\lambda$ или $\mu$ к $\mathbf{E} X_{1}$ приводит, вообще говоря, к нарушению этого соотношения.

Нашей основной целью является доказательство того, что для определенных классов плотностей $p$, удовлетворяющих условию $L(\lambda)=\infty$, соотношение (1.4) имеет место равномерно по всем $x \geqslant n \mu$.

Аналогичные задачи решались в [2], [4], [14], [15] и [16] (подробнее см. п. 2). Здесь же лишь отметим, что утверждения из [2], близкие по формулировкам к некоторым результатам настоящей работы, являются скорее правдоподобными предположениями, а не строго доказанными фактами (в качестве комментария к вышесказанному см. замечание в конце доказательства теоремы 2 А в п. 3).

2. Результаты. Пусть

$$
p(x) \sim e^{-\lambda x} x^{\rho-1} l(x), \quad x \rightarrow \infty
$$

где постоянные $\lambda, \rho>0$, функция $l(x)$ медленно меняется на бесконечности.

Очевидно, что при условии (2.1) выполняется соотношение (1.1) и $L(\lambda)=\infty$.

Теорема 1А. Если выполняется условие (2.1), то при любом $m \geqslant 1$

$$
p^{* m}(x)=c(m) e^{-\lambda x} x^{m \rho-1} l^{m}(x)(1+o(1)), \quad x \rightarrow \infty,
$$

әде $c(m)=\Gamma^{m}(\rho) / \Gamma(m \rho)$.

Теорема 2А. Пусть выполняются условия (2.1) и (1.3). Тогда соотношение (1.4) справедливо равномерно по всем $x \geqslant n \mu$, где постоянная $\mu>\mathbf{E} X_{1}$.

3 а м е ч а н и е 1 . Теорема 2 А является новой лишь тогда, когда $x>n m\left(\lambda_{0}\right)$ (см. (1.4), (1.5)).

3 а м е ч а н и е 2 . Коэффициенты $a_{j}(h)$ из соотношения (1.4) определяются равенством

$$
a_{j}(h)=\sum \frac{(2 j+2 l) !}{(j+l) ! 2^{j+l}}(-1)^{j+l} \prod_{m=1}^{2 j} \frac{1}{k_{m} !}\left(\frac{\lambda_{m+2}(h)}{(m+2) !}\right)^{k_{m}},
$$


где суммирование производится по всем целым неотрицательным решениям $\left(k_{1}, \ldots, k_{2 j}\right)$ уравнения $1 \cdot k_{1}+\cdots+2 j k_{2 j}=2 j$, a $l=k_{1}+\cdots+k_{2 j}$. Здесь (см. (1.2)) $\lambda_{m+2}(h)=(\ln L(h))^{(m+2)} / \sigma^{m+2}(h)$. Так, $a_{1}(h)=\frac{1}{8} \lambda_{4}(h)-$ $\frac{5}{24}\left(\lambda_{3}(h)\right)^{2}$.

Отметим, что в случае выполнения условия (2.1)

$$
\begin{aligned}
\lambda_{k}(h) & \sim(k-1) ! \rho^{1-k / 2} \quad \text { и, в частности, } \\
a_{1}(h) & \sim-\frac{1}{12} \rho^{-1}, \quad h \uparrow \lambda .
\end{aligned}
$$

Пусть $S_{n}=X_{1}+\cdots+X_{n}$.

Следствие 1А. Если выполняются условия (2.1), (1.3), то

$$
\mathbf{P}\left\{x \leqslant S_{n}<x+y\right\}=\frac{1-e^{-h y}}{h \sigma(h) \sqrt{2 \pi n}} L^{n}(h) e^{-h x}\left(1+O\left(\frac{1}{n}\right)\right),
$$

при $n \rightarrow \infty$, равномерно по $x \geqslant n \mu u y>0$, где $h$ удовлетворяет уравнению (1.5), а постоянная $\mu>\mathbf{E} X_{1}$.

Из (2.4) следует, что при $n \rightarrow \infty$

$$
\begin{aligned}
\mathbf{P}\left\{S_{n} \geqslant x\right\} & =\frac{1}{h \sigma(h) \sqrt{2 \pi n}} L^{n}(h) e^{-h x}\left(1+O\left(\frac{1}{n}\right)\right), \\
p^{* n}(x) & =\frac{1}{\sigma(h) \sqrt{2 \pi n}} L^{n}(h) e^{-h x}\left(1+O\left(\frac{1}{n}\right)\right)
\end{aligned}
$$

равномерно по $x \geqslant n \mu$ (на самом деле, соотношения (2.4) и (2.6) равносильны).

Обращаем внимание на то, что, в отличие от аналогичных хорошо известных равенств (см. [3], [5]-[7]), соотношение (2.5) справедливо при произвольно больших $x / n$.

Следует также сказать, что решетчатые аналоги соотношений (2.5) и (2.6) содержатся в [16, теорема 2 (условие $(\mathrm{R}))]$. Там же отмечено, что аналогичные результаты справедливы и в абсолютно непрерывном случае.

Следствие 2А. Пусть $\tau_{n}$ стремится $\kappa$ бесконечности как угодно медленно. Если выполняются условия (2.1) и (1.3), то при $n \rightarrow \infty$ равномерно по $t \geqslant \tau_{n}$

$$
\begin{aligned}
-\frac{1}{n} \ln p^{* n}(t n) & =-\frac{1}{n} \ln \mathbf{P}\left\{S_{n} \geqslant t n\right\}+o(1)=\lambda t-\nu_{n}\left(\frac{t}{\rho}\right)+o(1) \sim \lambda t, \\
p^{* n}(t n) & =\frac{1}{\sigma(h) \sqrt{2 \pi n}} e^{n(\ln L(h)-h t)}\left(1-\frac{1+o(1)}{12 \rho n}\right),
\end{aligned}
$$

где $\nu_{n}(y)=\ln \left(e^{\lambda y} y p(y)\right)+\rho+\ln \Gamma(\rho)-\frac{1}{n} \ln y, h=h(t)-$ решение уравнения $m(h)=t$. 
П р и м е $\mathrm{p}$ А. Пусть $p(x)=e^{x} /\left(1+e^{x}\right)^{2},-\infty<x<\infty$ (т.е. является плотностью логистического распределения (см. [12])). Тогда при $\lambda=\rho=1, l(x) \equiv 1$ выполняется условие $(2.1)$ и из соотношений $(2.8)$, (2.5) следует (детали см. в п. 5), что при $n \rightarrow \infty$

$$
\begin{aligned}
p^{* n}(t n) & =\frac{t^{n-1}}{\sqrt{2 \pi n}} e^{-n t(1-u(1 / t))}\left(1+v_{1}\left(\frac{1}{t}\right)-\frac{1+o(1)}{12 n}\right), \\
\mathbf{P}\left\{S_{n} \geqslant t n\right\} & =\frac{t^{n-1}}{\sqrt{2 \pi n}} e^{-n t(1-u(1 / t))}\left(1+v_{2}\left(\frac{1}{t}\right)+O\left(\frac{1}{n}\right)\right)
\end{aligned}
$$

равномерно по $t \geqslant \tau_{n}$, где $\tau_{n}$ стремится к бесконечности как угодно медленно, а функции $u(x)$ и $v_{1}(x), v_{2}(x)$ в окрестности нуля можно представить в виде равномерно сходящихся рядов по степеням $x$ с известными коэффициентами. Так,

$$
\begin{gathered}
u(x)=x-x^{2}+\left(\frac{\pi^{2}}{6}-1\right) x^{3}+\cdots, \\
v_{1}(x)=x-\frac{\pi^{2}-5}{2} x^{2}+\cdots, \quad v_{2}(x)=2 x+\frac{11-\pi^{2}}{2} x^{2}+\cdots .
\end{gathered}
$$

Теоремы $1 \mathrm{~A}, 2 \mathrm{~A}$ и следствие $1 \mathrm{~A}$ будут доказаны в п. 3 . Там же содержится основная схема доказательств.

Далее рассмотрим еще один класс плотностей.

Пусть $\xi=\xi(x)$ - некоторая положительная функция, удовлетворяющая условию

$$
\limsup _{x \rightarrow \infty}\left|\xi^{\prime}(x)\right|<\infty
$$

Мы будем предполагать, что

$$
p(x) \sim u(x) e^{-g(x)}, \quad x \rightarrow \infty,
$$

где функция $g$ удовлетворяет условиям

$$
\begin{gathered}
\limsup _{x \rightarrow \infty} \frac{g(x)}{x}=\lambda>0, \\
\xi^{2} g^{\prime \prime}(x) \rightarrow \infty
\end{gathered}
$$

(можно показать, что при этих предположениях, так же, как в предыдущем случае, выполняется соотношение $(1.1)$ и $L(\lambda)=\infty)$.

Помимо этого потребуем, чтобы при $x \rightarrow \infty$

$$
g^{\prime \prime}(x+o(\xi))=(1+o(1)) g^{\prime \prime}(x), \quad u(x+o(\xi))=(1+o(1)) u(x) .
$$

Заметим, что из условий (2.9) и (2.13) следует соотношение

$$
\left(c e^{k \omega(x)}\right)^{-1} \leqslant g^{\prime \prime}(x), u(x) \leqslant c e^{k \omega(x)}, \quad x \geqslant x_{0} \quad(\exists c, k>0),
$$

в котором $\omega(x)=\int_{x_{0}}^{x} d u / \xi(u)$. 
Можно положить $\xi(x)=x$, и такой выбор при $\lambda<\infty$ (см. (2.11)) выглядит вполне естественным. Он, однако, не позволяет рассматривать достаточно быстро убывающие вероятностные плотности (см. (2.14) с $\omega(x)=\ln x)$, что при $\lambda=\infty$ является сушественным ограничением. В типичных случаях, вообще говоря, $\xi(x) \sim g^{\prime \prime}(x) /\left|g^{\prime \prime \prime}(x)\right|$. Так, если $g(x)=e^{x}$, то по этому правилу $\xi(x)=1$, и условия $(2.12),(2.13)$ удовлетворяются для широкого спектра функций $u(x)$; если $g(x)=\exp \left\{e^{x}\right\}$, то $\xi(x)=e^{-x}$, и т.д.

Отметим, что аналогичный, заметно более узкий, класс плотностей был введен в [2], и что при $u(x)=\sqrt{g^{\prime \prime}(x) /(2 \pi)}$ и $\xi(x)=v(x) / \sqrt{g^{\prime \prime}(x)}$, где функция $v(x)$ достаточно медленно стремится к бесконечности, удовлетворяются условия на вероятностные плотности, асимптотическое поведение сверток которых изучалось в [4].

Приведем результаты.

Теорема 1В. Если выполняются условия (2.10)-(2.13), то при любом $m \geqslant 1$

$$
p^{* m}(x)=\frac{1}{\sqrt{m}}\left(\frac{2 \pi}{g^{\prime \prime}(x / m)}\right)^{(m-1) / 2} p^{m}\left(\frac{x}{m}\right)(1+o(1)),
$$

когда $x \rightarrow \infty$.

Теорема 2В. Пусть выполняются условия (2.10)-(2.13) и (1.3). Тогда имеет место утверждение теоремь $2 \mathrm{~A}$. При этом (см. замечание 2) $\lim _{h \uparrow \lambda} \lambda_{k}(h)=0$ и, следовательно, $\lim _{h \uparrow \lambda} a_{j}(h)=0$.

Если (см. (2.11)) $\lambda<\infty$, то условия на плотность $p(x)$ можно равносильно переформулировать в более естественной форме, введя функцию $s(x)=\lambda x-g(x)$. В этом случае вместо соотношений (2.10)-(2.13) имеем

$$
p(x) \sim u(x) e^{-\lambda x+s(x)}, \quad x \rightarrow \infty,
$$

причем $\lim \inf _{x \rightarrow \infty} s(x) / x=0$ и при $x \rightarrow \infty$

$$
\begin{aligned}
& \xi^{2} s^{\prime \prime}(x) \rightarrow-\infty, s^{\prime \prime}(x+o(\xi)) \\
& u(x+o(\xi))=(1+o(1)) s^{\prime \prime}(x), \\
&u(1)) u(x) .
\end{aligned}
$$

Следствие 1В. Пусть выполняются условия теоремь 2В. Тогда имеет место утверждение следствия $1 \mathrm{~A}$ и, в частности, справедливы соотношения (2.5), (2.6).

Заметим, что теорема 2 (условие $(\mathrm{S}))$ из [16] содержит решетчатый аналог соотношений $(2.5)$ и (2.6) из следствия $1 \mathrm{~B}$ при $\lambda<\infty, u(x)=1$ и $\xi(x)=x$. При этом само условие (S) является более ограничительным, нежели условие (2.16) (см. [16, определение 1 и начало доказательства леммы 1]).

Следствие 2В. Пусть $\tau_{n}$ стремится $\kappa$ бесконечности как угодно медленно и выпслняются условия (2.10)-(2.13), (1.3). Тогда при $n \rightarrow \infty$ 
равномерно по $t \geqslant \tau_{n}$

$$
\begin{gathered}
-\frac{1}{n} \ln p^{* n}(t n)=-\frac{1}{n} \ln \mathbf{P}\left\{S_{n} \geqslant t n\right\}-\frac{1}{n} \ln g^{\prime}(t)+o(1) \\
=-\ln p(t)+\frac{1}{2}\left(1-\frac{1}{n}\right) \ln g^{\prime \prime}(t)-\ln \sqrt{2 \pi}+o(1) \sim g(t), \\
p^{* n}(t n)=\frac{1}{\sigma(h) \sqrt{2 \pi n}} e^{n(\ln L(h)-h t)}\left(1+o\left(\frac{1}{n}\right)\right),
\end{gathered}
$$

где $h=h(t)$ - решение уравнения $m(h)=t$.

Заметим, что если в условии (2.11) $\lambda \neq \infty$, то, так же, как в соотношении (2.7), из (2.17) вытекает

$$
-\frac{1}{n} \ln p^{* n}(t n)=-\frac{1}{n} \ln \mathbf{P}\left\{S_{n} \geqslant t n\right\}+o(1) \sim \lambda t, \quad t \geqslant \tau_{n}, \quad n \rightarrow \infty .
$$

П р и м е р В. Пусть $p(x)=e^{x-e^{x}}, 0 \leqslant x<\infty$ (т.е. является плотностью двойного показательного распределения, или распределения Гумбеля, см. [12]). Тогда при $u(x)=g(x)=e^{x}, \xi(x) \equiv 1$ выполняются условия (2.9)-(2.13) и (см. п. 5)

$$
\begin{gathered}
p^{* n}(t n)=(2 \pi)^{(n-1) / 2} e^{(n+1) t / 2-n e^{t}\left(1-u\left(e^{-t}\right)\right)}\left(1+v_{1}\left(e^{-t}\right)+(1+o(1)) \frac{e^{-t}}{24 n}\right), \\
\mathbf{P}\left\{S_{n} \geqslant t n\right\}=(2 \pi)^{(n-1) / 2} e^{(n-1) t / 2-n e^{t}\left(1-u\left(e^{-t}\right)\right)}\left(1+v_{2}\left(e^{-t}\right)+O\left(\frac{1}{n}\right)\right)
\end{gathered}
$$

при $n \rightarrow \infty$ равномерно по $t \geqslant \tau_{n}$, где $\tau_{n}$ стремится к бесконечности как угодно медленно, а функции $u(x), v_{1}(x)$ и $v_{2}(x)$ имеют асимптотическое представление в виде рядов при $x \rightarrow 0$

$$
u(x) \sim \frac{1}{24} x+\cdots, \quad v_{1}(x) \sim \frac{5}{48} x^{2}+\cdots, \quad v_{2}(x) \sim \frac{1}{2} x+\frac{19}{48} x^{2}+\cdots .
$$

Теоремы 1В, 2В и их следствия будут доказаны в п. 4.

В заключение рассмотрим два класса плотностей $p(x)$, носитель которых, в отличие от уже рассмотренных случаев, ограничен справа постоянной $r$. В этом случае условие (1.1) выполняется и $\lambda=\infty$.

Ниже будем предполагать, что $\sup \operatorname{supp} p(x)=r=0$. Это не умаляет общности, поскольку всегда может быть достигнуто заменой $X_{i}$ на $X_{i}-r$. Сначала приведем аналоги теорем $1 \mathrm{~A}$ и $2 \mathrm{~A}$.

Пусть

$$
p(x) \sim|x|^{\rho-1} l(|x|), \quad x \rightarrow-0,
$$

где постоянная $\rho>0$, а функция $l(|x|)$ медленно меняется в нуле.

Теорема 1C. Если выполняется условие (2.19), то при любом $m \geqslant 1$

$$
p^{* m}(x)=c(m)|x|^{m \rho-1} l^{m}(|x|)(1+o(1)), \quad x \rightarrow-0,
$$

где $c(m)$ определено в теореме $1 \mathrm{~A}$. 
Теорема 2С. Пусть выполняются условия (2.19) и (1.3). Тогда равенство (1.4) справедливо равномерно по всем $0>x \geqslant n \mu$, где $\mu>\mathbf{E} X_{1}$. Соотношение (2.3) также выполняется.

Следствие 1С. Если выполняются условия (2.19) и (1.3), то соотношение (2.4) справедливо равномерно по $0>x \geqslant n \mu$ u $0<y<|x|$.

Из следствия $1 \mathrm{C}$ следует, что соотношения (2.5) и (2.6) при $n \rightarrow \infty$ имеют место равномерно по $0>x \geqslant n \mu$. Таким образом, несколько уточнена теорема 1 из [15], в которой аналогичные результаты были получены в предположении (1.3) при $m=1$ и с оценкой погрешности $O(1 / \sqrt{n})$.

Заметим, что справедливость равенства (2.5) (с $o(1)$ вместо $O(1 / n))$ была установлена при более слабых, нежели (2.19), предположениях в [7, теорема В], где вместо (2.19) требовалось, чтобы $\mathbf{P}\left\{X_{1}<x\right\} \sim|x|^{\rho} l(|x|)$, $x \rightarrow-0$.

Следствие 2С. Пусть $\tau_{n}$ стремится $\kappa$ нулю как угодно медленно. Если выполняются условия (2.19) и (1.3), то при $n \rightarrow \infty$ равномерно по $0<t \leqslant \tau_{n}$ выполняется соотношение $(2.8)$, где $h=h(t)-$ решение уравнения $m(h)=t, u$, кроме того,

$$
\begin{aligned}
-\frac{1}{n} \ln \mathbf{P}\left\{S_{n} \geqslant-t n\right\} & =-\frac{1}{n} \ln p^{* n}(-t n)-\frac{\ln t}{n}+o(1) \\
& =\rho-\ln \Gamma(\rho)-\ln \left(\frac{t}{\rho} p\left(\frac{t}{\rho}\right)\right)+o(1) \sim \rho \ln \frac{\rho}{t} .
\end{aligned}
$$

П р и м е р С. Пусть случайная величина $X_{1}$ имеет гаммараспределение с параметром $\rho>0$, или $p(x)=e^{-x} x^{\rho-1} / \Gamma(\rho), 0 \leqslant x<\infty$. Если $0<t_{n} \rightarrow 0$, то равномерно по $t$ при $n \rightarrow \infty$

$$
\begin{aligned}
\mathbf{P}\left\{0<S_{n} \leqslant t n\right\} & =\sqrt{\frac{\rho}{2 \pi n}}(\rho-t)^{-1}\left(\frac{t}{\rho}\right)^{n \rho} e^{n(\rho-t)}\left(1+O\left(\frac{1}{n}\right)\right), \\
p^{* n}(t n) & =\sqrt{\frac{\rho n}{2 \pi}}\left(\frac{\rho n}{e}\right)^{\rho n} e^{-n t}(n t)^{\rho n-1}(1+v(\rho n)),
\end{aligned}
$$

где $v(x) \sim \sum_{j \geqslant 1} \alpha_{j} x^{-j}, x \rightarrow \infty\left(\alpha_{1}=-\frac{1}{12}\right)$.

Отметим, что из второго равенства и соотношения $p^{* n}(x)=$ $e^{-x} x^{n \rho-1} / \Gamma(n \rho), x \geqslant 0$, следует формула

$$
\Gamma(s) \sim \sqrt{\frac{2 \pi}{s}}\left(\frac{s}{e}\right)^{s}\left(1+\sum_{j \geqslant 1} \alpha_{j} s^{-j}\right)^{-1}, \quad s \rightarrow \infty,
$$

в которой коэффициенты $\left\{\alpha_{j}\right\}$ явно определены.

Пример С вытекает из теоремы $2 \mathrm{C}$ и следствия $1 \mathrm{C}$ (также см. п. 5).

Нижеследующие утверждения по формулировкам близки к теоремам $1 \mathrm{~B}$ и $2 \mathrm{~B}$. 
Пусть положительная функция $\xi=\xi(x)$ определена при всех достаточно малых $x<0$, причем

$$
\lim _{x \rightarrow-0} \xi(x)=0 \quad \text { и } \quad \limsup _{x \rightarrow-0}\left|\xi^{\prime}(x)\right|<\infty .
$$

Будем предполагать, что

$$
p(x) \sim u(x) e^{-g(x)}, \quad x \rightarrow-0,
$$

где функции $u(x)$ и $g(x)$ при $x \rightarrow-0$ удовлетворяют условиям (2.13) и

$$
g(x) \rightarrow \infty, \quad \xi^{2} g^{\prime \prime}(x) \rightarrow \infty .
$$

Теорема 1D. Если выполняются условия (2.21)-(2.23), то для любого $m \geqslant 1$ при $x \rightarrow-0$ имеет место соотношение (2.15).

Заметим, что теорема $1 \mathrm{D}$ при $u(x)=\sqrt{g^{\prime \prime}(x) /(2 \pi)}$ и $\xi(x)=x$ близка к теореме 1 из [14].

Теорема 2D. Пусть выполняются условия (2.21)-(2.23) и (1.3). Тогда справедливо заключение теоремы $2 \mathrm{~B}($ при $\lambda=\infty)$ и, в частности, имеют место соотношения (2.4) $(0>x>n \mu u 0<y<|x|),(2.5)$, (2.6) $(0>x>n \mu)$ и (2.17), (2.18), причем последние два соотношения выполняются равномерно по $t,-\varepsilon_{n}<t<0$, где положительное $\varepsilon_{n}$ стремится $к$ нулю как угодно медленно.

3. Доказательства теорем 1 А и 2 . Начнем с доказательства равенства (2.2), которое проведем по индукции. Пусть $\eta$ достаточно медленно стремится к бесконечности при $x \rightarrow \infty$ (например, $\eta=\sqrt{\ln x}$ ). Имеем

$$
\begin{aligned}
p^{*(m+1)}(x) & =\left(\int_{-\infty}^{\eta}+\int_{\eta}^{x-\eta}+\int_{x-\eta}^{\infty}\right) p(x-y) p^{* m}(y) d y \\
& =I_{1}+I_{2}+I_{3} .
\end{aligned}
$$

Применяя равенство (2.2) и имея в виду, что функция $l(x)$ медленно меняется (см. [8, гл. VIII]), легко получим при $x \rightarrow \infty$

$$
\begin{aligned}
I_{2} & \sim c(m) e^{-\lambda x} x^{(m+1) \rho-1} \int_{\eta / x}^{1-\eta / x}(1-t)^{\rho-1} l(x(1-t)) t^{m \rho-1} l^{m}(t x) d t \\
& \sim c(m) e^{-\lambda x} l^{m+1}(x) x^{(m+1) \rho-1} \int_{0}^{1}(1-t)^{m \rho-1} t^{\rho-1} d t
\end{aligned}
$$

(напоминаем, что $\max _{\eta / x \leqslant u \leqslant 1} l(u x) / l(x) \leqslant 2 u^{-\varepsilon}, 0<\varepsilon<\rho$, при всех достаточно больших $x$, и этот факт позволяет подходяшим образом оценить интеграл в концевых точках).

Далее,

$$
\begin{aligned}
& I_{3} \leqslant \sup _{t \geqslant x-\eta} p^{* m}(t) \sim c(m) e^{\lambda \eta} e^{-\lambda x} x^{m \rho-1} l^{m}(x), \quad x \rightarrow \infty, \\
& I_{1} \leqslant \sup _{t \geqslant x-\eta} p(t) \sim e^{\lambda \eta} e^{-\lambda x} x^{\rho-1} l(x), \quad x \rightarrow \infty
\end{aligned}
$$


откуда следует, что

$$
I_{1}+I_{3}=o(1) e^{-\lambda x} x^{(m+1) \rho-1} l^{m+1}(x), \quad x \rightarrow \infty .
$$

Для проверки индукционного перехода пользуемся соотношениями (3.1)-(3.3) и тем, что $c(m) B(m \rho, \rho)=c(m+1)$. Теорема 1А доказана.

Теперь проверим справедливость теоремы 2А. Имеем (см., например, [2]): при $0<h<\lambda$ и любом $x$

$$
p^{* n}(x)=L^{n}(h) e^{-h x} p_{h}^{* n}(x),
$$

где $p_{h}(x)=e^{h x} p(x) / L(h)$ - плотность случайной величины $X^{(h)}$, имеющей сопряженное с $V$ распределение $V^{(h)}$. Хорошо известно, что $m(h)=\mathbf{E} X^{(h)}, \sigma^{2}(h)=\mathbf{D} X^{(h)}$.

Обозначим $f_{h}(t)$ характеристическую функцию случайной величины $Y_{h}=\left(X^{(h)}-m(h)\right) / \sigma(h)$.

Пусть постоянная $m$ взята из условия (1.3). Покажем, что

$$
\sup _{\lambda_{0} \leqslant h<\lambda} \int_{-\infty}^{\infty}\left|f_{h}(t)\right|^{2 m} d t<\infty
$$

где положительная постоянная $\lambda_{0}$ достаточно близка к $\lambda$.

Положим $\delta=\lambda-h>0$. Пользуясь свойствами медленно меняющихся функций, несложно установить (см. также $[2$, теорема 7.1$]$ ), что при $\delta \rightarrow 0$

$$
L(h) \sim \Gamma(\rho) \delta^{-\rho} l \delta^{-1}, \quad m(h) \sim \rho \delta^{-1}, \quad \sigma^{2}(h) \sim \rho \delta^{-2} .
$$

Докажем теперь, что при условиях (2.1) и (1.3)

$$
\sigma(h) \sup _{x} p_{h}^{* m}(x) \leqslant c_{1}, \quad \varepsilon \leqslant h<\lambda
$$

(здесь и в дальнейшем положительные постоянные $c_{i}, i \geqslant 1$, не зависят от $h)$.

По (3.4) $p_{h}^{* m}(x)=e^{h x} p^{* m}(x) / L^{m}(h)$. Считая без потери общности, что $m \rho>1$, и имея в виду (1.3) и (3.6), получим при достаточно большом $x_{0}$

$$
\sigma(h) \sup _{x<x_{0}} p_{h}^{* m}(x) \leqslant c_{2} e^{h x_{0}} \sup _{\varepsilon \leqslant h<\lambda} \frac{\sigma(h)}{L^{m}(h)}<\infty .
$$

Аналогично, по (2.2)

$$
\sigma(h) \sup _{x \geqslant x_{0}} p_{h}^{* m}(x) \leqslant 2 c(m) \frac{\sigma(h)}{L^{m}(h)} \sup _{x \geqslant x_{0}} e^{-\delta x} x^{m \rho-1} l^{m}(x)=2 I .
$$

Поскольку $0<\delta<\lambda-\lambda_{0}$, а $\lambda-\lambda_{0}$ достаточно мало, то по (3.6)

$$
I<\frac{2 \sqrt{\rho}}{\Gamma(m \rho)} \sup _{x \geqslant x_{0}} e^{-\delta x}(\delta x)^{m \rho-1}\left(\frac{l(x)}{l(1 / \delta)}\right)^{m} \leqslant c_{3}
$$


(напоминаем, что по свойствам медленно меняющихся функций $l(x) / l(1 / \delta) \leqslant 2\left((\delta x)^{\eta}+(\delta x)^{-\eta}\right), x \geqslant x_{0}, 0<\delta<\delta_{0}$, где постоянная $\eta \in(0, m \rho-1)$, если $x_{0}$ и $1 / \delta_{0}$ достаточно велики $)$.

Итак, соотношение (3.7) доказано.

Из (3.7) вытекает, что плотность $p_{h}^{* m}(x)$ интегрируема с квадратом и, следовательно,

$$
\int_{-\infty}^{\infty}\left|f_{h}^{m}(t)\right|^{2} d t=2 \pi \int_{-\infty}^{\infty}\left(\sigma(h) p_{h}^{* m}(x \sigma(h))\right)^{2} d x \leqslant 2 \pi \sigma(h) \sup _{x} p_{h}^{* m}(x) \leqslant c_{4},
$$

что и доказывает соотношение (3.5).

Пусть теперь $h$ является решением уравнения (1.5). Пользуясь условием (3.5) и формулой обращения для плотностей, несложно установить, что при $n \geqslant 2 m$

$$
p_{h}^{* n}(x)=\frac{1}{2 \pi \sigma(h) \sqrt{n}} \int_{-\infty}^{\infty} f_{h}^{n}\left(\frac{t}{\sqrt{n}}\right) d t .
$$

Функция $f_{h}^{n}(t / \sqrt{n})$ является характеристической функцией нормированной суммы $n$ независимых случайных величин, каждая из которых распределена так же, как стандартизованная случайная величина $Y_{h}$. Поэтому для исследования интеграла $\int_{-\infty}^{\infty} f_{h}^{n}(t / \sqrt{n}) d t$ можно применить хорошо разработанную технику доказательства локальных предельных теорем для плотностей $[9$, гл. VII]. и 4])

Положим $\alpha_{k}=\alpha_{k}(h)=\mathbf{E} Y_{h}^{k}, k \geqslant 3$. Имеем (см. [9, гл. VI, леммы 2

$$
\begin{aligned}
& \left|\int_{-\infty}^{\infty}\left(f_{h}^{n}\left(\frac{t}{\sqrt{n}}\right)-e^{-t^{2} / 2}\left(1+\sum_{\nu=1}^{2 s-1} P_{\nu}(i t) n^{-\nu / 2}\right)\right) d t\right| \\
& \quad \leqslant C(s) \alpha_{2 s+2} n^{-s}+\sqrt{n} \int_{|t|>\gamma_{s}}\left|f_{h}^{n}(t)\right| d t
\end{aligned}
$$

где постоянная $C(s)$ зависит лишь от $s$, многочлены $P_{\nu}(i t)$ определены в $[9$, гл. VI, $(1.12)], \gamma_{s}=\left(\alpha_{2 s+2}\right)^{-1 /(2 s)}$. При этом $([9$, гл. VI, $(1.10)]$ и замечание 2)

$$
\int_{-\infty}^{\infty} e^{-t^{2} / 2}\left(1+\sum_{\nu=1}^{2 s-1} P_{\nu}(i t) n^{-\nu / 2}\right) d t=\sum_{j=1}^{s-1} q_{2 j}(0) n^{-j}=\sum_{j=1}^{s-1} a_{j}(h) n^{-j}
$$

в соответствии с определением функций $q_{2 j}(x)$, поскольку величины $\lambda_{k}(h)$ совпадают с кумулянтами случайной величины $Y_{h}$ того же порядка.

Из (1.1) и [2, теорема 7.1] следует, что

$$
\sup _{\lambda_{0} \leqslant h<\lambda} \alpha_{2 s+2}(h) \leqslant c_{5} \quad \text { или } \quad \gamma_{s} \leqslant c_{6} ;
$$

там же приводится (содержашая неточность) формула для $\lambda_{k}(h)$ из $(2.3)$. 
Далее,

$$
\int_{|t|>\gamma_{s}}\left|f_{h}^{n}(t)\right| d t \leqslant \sup _{|t|>\gamma_{s}}\left|f_{h}^{m}(t)\right|^{n / m-2} \int_{-\infty}^{\infty}\left|f_{h}^{m}(t)\right|^{2} d t .
$$

Нам понадобится одно вспомогательное утверждение, которое можно найти в $[10]$ (см. также $[11,(16)])$.

Лемма 1. Пусть $q(x), f(t)$ и $\sigma^{2}$ - соответственно плотность, характеристическая функиия и дисперсия некоторой случайной величиньl, $c=\sup _{x} q(x)$. Тогда

$$
|f(t)| \leqslant 1-\frac{1}{96} \frac{t^{2}}{c^{2}(\pi+2 \sigma|t|)^{2}} \quad \forall t .
$$

Из леммы 1 и оценки (3.7) следует, что

$$
\left|f_{h}^{m}(t)\right| \leqslant 1-\frac{1}{96} \frac{t^{2}}{c_{1}^{2}(\pi+2 m|t|)^{2}} \leqslant e^{-\eta}, \quad|t| \geqslant \frac{1}{c_{6}},
$$

где положительное $\eta$ не зависит от $t$. Таким образом, при всех достаточно больших $n$

$$
\sup _{\lambda_{0} \leqslant h<\lambda} \int_{|t|>\gamma_{s}}\left|f_{h}^{n}(t)\right| d t \leqslant c_{7} n^{-s-1} .
$$

Отсюда, из (3.4) и (3.9)-(3.12) следуют теорема 2 А и замечание 2.

3 а м е ч а н и е. В [2] отсутствует оценка (3.13) либо какой-нибудь ее аналог. То же самое можно сказать об условии (1.3).

Теперь докажем следствие $1 \mathrm{~A}$. Из теоремы $2 \mathrm{~A}$ при $s=1$ следует соотношение (2.6), из которого, в свою очередь, вытекает равенство

$$
\mathbf{P}\left\{x \leqslant S_{n}<x+y\right\}=\frac{1+O(1 / n)}{\sqrt{2 \pi n}} \int_{x}^{x+y} \frac{1}{\sigma(h)} e^{\kappa(h)} d u, \quad n \rightarrow \infty,
$$

равномерно по $x \geqslant n m(\varepsilon)$ и $y>0$, где $\kappa(h)=n \ln L(h)-h u, h=h(u / n)$ (см. (1.5)), $\varepsilon$ - некоторая положительная постоянная.

Очевидно,

$$
\kappa_{u}^{\prime}=-h, \quad \kappa_{u u}^{\prime \prime}=-h_{u}^{\prime}=-\frac{1}{n \sigma^{2}(h)} .
$$

Положим $\widehat{h}=h(x / n)$. Воспользовавшись равенствами (3.15), имеем

$$
\begin{gathered}
h-\widehat{h}=(u-x)\left(n \sigma^{2}(\tilde{h})\right)^{-1}, \\
\sigma^{2}(h)-\sigma^{2}(\widehat{h})=(u-x)\left(n \sigma^{2}(\widetilde{h})\right)^{-1}\left(\sigma^{2}(\widetilde{h})\right)^{\prime}, \\
\kappa(h)=\kappa(\widehat{h})-(u-x) \widehat{h}-\frac{1}{2}(u-x)^{2}\left(n \sigma^{2}(\widetilde{h})\right)^{-1},
\end{gathered}
$$

где $\tilde{h} \in(\widehat{h}, h)$, причем $\tilde{h}$ в разных формулах могут не совпадать. 
Далее (см. (1.2), (3.6) и (2.3))

$$
\frac{1}{\sigma^{3}(h)}\left(\sigma^{2}(h)\right)^{\prime} \sim 2 \rho, \quad h \uparrow \lambda .
$$

Пусть $\delta=\lambda-h, \widehat{\delta}=\lambda-\widehat{h}$. Из (2.1), (3.6) и (3.18) следует, что

$$
0<\inf \delta^{2} \sigma^{2}(h) \leqslant \sup \delta^{2} \sigma^{2}(h)<\infty, \quad \sup \delta^{3}\left|\left(\sigma^{2}(h)\right)^{\prime}\right|<\infty,
$$

где inf и sup берутся по всем $h \in[\varepsilon, \lambda)$.

Пусть $0<y \leqslant y_{n}=\sqrt{n} \sigma(\widehat{h})$. Функция $h=h(u / n)$ возрастает (см. (3.15)). Поэтому из (3.16), (3.18) и (3.19) следует, что при $u \in(x, x+y)$

$$
0 \leqslant h-\widehat{h}=\widehat{\delta}-\delta \leqslant c_{1} y_{n} \frac{\widehat{\delta}^{2}}{n} \leqslant c_{2} \frac{1}{\sqrt{n}} \widehat{\delta}
$$

и при всех достаточно больших $n$

$$
\frac{1}{\sigma(h)}=\frac{1}{\sigma(\widehat{h})}\left(1+\frac{\sigma^{2}(h)-\sigma^{2}(\widehat{h})}{\sigma(h)(\sigma(\widehat{h})+\sigma(h))}\right)=\frac{1}{\sigma(\widehat{h})}\left(1+\theta_{0} \frac{u-x}{n \sigma(\widehat{h})}\right)
$$

(здесь и ниже посредством $\theta_{i}, i=0,1, \ldots$, обозначены некоторые переменные такие, что $\left.\left|\theta_{i}\right| \leqslant c_{i}\right)$.

Из (3.17) и (3.20) при $0<y \leqslant y_{n}$ и $\tau_{n}=\sqrt{n} \widehat{h} \sigma(\widehat{h})$ следует, что

$$
\begin{aligned}
\int_{x}^{x+y} \frac{1}{\sigma(h)} e^{\kappa(h)} d u= & e^{\kappa(\widehat{h})} \frac{1}{\widehat{h} \sigma(\widehat{h})} \int_{0}^{y \widehat{h}}\left(1+\theta_{1} \frac{t}{\tau_{n} \sqrt{n}}\right) \\
& \times \exp \left\{-t-\frac{1}{2}\left(\frac{\theta_{2}}{\tau_{n}}\right)^{2} t^{2}\right\} d t .
\end{aligned}
$$

Обозначим интеграл в правой части (3.21) через $I$. Имеем

$$
I=I_{1}+\frac{\theta_{3}}{\tau_{n} \sqrt{n}} I_{2}
$$

где

$$
I_{1}=\int_{0}^{y \widehat{h}} \exp \left\{-t-\frac{1}{2}\left(\frac{\theta_{2}}{\tau_{n}}\right)^{2} t^{2}\right\} d t, \quad I_{2}=\int_{0}^{y \widehat{h}} t e^{-t} d t .
$$

Лемма 2. При любых $\beta, \gamma>0$

$$
1-e^{-\beta}>\int_{0}^{\beta} e^{-t-\frac{1}{2} \gamma t^{2}} d t>\left(1-e^{-\beta}\right)(1-\gamma) .
$$

Для доказательства применим в интеграле неравенство $1-\frac{1}{2} \gamma t^{2} \leqslant$ $e^{-\gamma t^{2} / 2} \leqslant 1$.

Из леммы 2 вытекает, что $I_{1}=\left(1-e^{-y \widehat{h}}\right)\left(1-\theta_{4} / \tau_{n}^{2}\right)$,

$$
I_{2}=\left(1-e^{-y \widehat{h}}\right)\left(1+\frac{y \widehat{h}}{e^{y \widehat{h}}-1}\right) \leqslant 2\left(1-e^{-y \widehat{h}}\right) .
$$


Отсюда и из (3.22) заключаем, что

$$
I=\left(1-e^{-y \widehat{h}}\right)\left(1+\frac{\theta_{5}}{n}\left(\frac{1}{\widehat{h} \sigma(\widehat{h})}+\left(\frac{1}{\widehat{h} \sigma(\widehat{h})}\right)^{2}\right)\right) .
$$

Учитывая (см. (3.6)), что $\inf _{\varepsilon \leqslant h<\lambda} h \sigma(h)>0$, из (3.21)-(3.23) и (3.14) получим утверждение следствия 1 А при $0<y \leqslant y_{n}$.

Пусть теперь $y>y_{n}$. Имеем

$$
\begin{aligned}
& \mathbf{P}\left\{x \leqslant S_{n}<x+y_{n}\right\} \leqslant \mathbf{P}\left\{x \leqslant S_{n}<x+y\right\} \\
& \quad \leqslant \mathbf{P}\left\{x \leqslant S_{n}<x+y_{n}\right\}+\mathbf{P}\left\{S_{n} \geqslant x+y_{n}\right\}=J_{1}+J_{2},
\end{aligned}
$$

причем (см. (2.4) при $\left.y=y_{n}\right)$ равномерно по $x \geqslant n m(\varepsilon)$

$$
J_{1}=\frac{1}{\tau_{n} \sqrt{2 \pi}} e^{\kappa(\widehat{h})}\left(1-e^{-\widehat{h} y_{n}}\right)\left(1+O\left(\frac{1}{n}\right)\right), \quad n \rightarrow \infty
$$

(напоминаем, что $\widehat{h}=h(x / n)$ и $\tau_{n}=\sqrt{n} \widehat{h} \sigma(\widehat{h})$ ).

Оценим $J_{2}$. Положим $\bar{h}=h\left(\left(x+y_{n}\right) / n\right)$. Применяя неравенство Чебышева и соотношение (3.17) при $h=\bar{h}$, а также имея в виду, что $\widehat{h} y_{n}=\tau_{n}$, получаем

$$
J_{2} \leqslant e^{\kappa(\bar{h})} \leqslant e^{\kappa(\widehat{h})-y_{n} \widehat{h}}=\frac{e^{\kappa(\widehat{h})}}{\tau_{n}} O\left(\frac{1}{\tau_{n}^{2}}\right), \quad n \rightarrow \infty .
$$

Из оценок (3.24)-(3.26) и того, что $\inf _{\varepsilon \leqslant h<\lambda}\left(\tau_{n} / \sqrt{n}\right)>0$ равномерно по $n$, следует равенство (2.4) при $y>y_{n}$. Следствие 1 А полностью доказано.

Соотношение (2.7) следствия $2 \mathrm{~A}$ вытекает из (2.5), (3.6) и (1.5), а (2.8) из (2.3) и теоремы 2 А при $s=2$.

\section{4. Доказательства теорем 1В и 2 В.}

Лемма 3. Пусть $x_{0}, t_{0}-$ некоторые положительные числа. Предположим, что при всех $x>x_{0}$ функчия $\xi(x)$ непрерывна слева $u$, кроме того,

$$
\xi=\xi(x)>0 \quad u \quad \frac{\xi(x+t \xi)}{\xi} \leqslant L(t), \quad t \in\left[0, t_{0}\right],
$$

әде $L(t), 0 \leqslant t \leqslant t_{0}$, - некоторая непрерьвная функиия. Если

$$
v(x+o(\xi))=(1+o(1)) v(x), \quad x \rightarrow \infty,
$$

то для любого $\varepsilon \in(0,1)$ найдутся постоянные $k>0 u \bar{x} \geqslant x_{0}$ такие, umo

$$
(1+\varepsilon)^{-(1+k(\omega(y)-\omega(x)))} \leqslant \frac{v(y)}{v(x)} \leqslant(1+\varepsilon)^{1+k(\omega(y)-\omega(x))} \quad \forall y, x: y \geqslant x \geqslant \bar{x} .
$$

Здесь

$$
\omega(x)=\int_{x_{0}}^{x} \frac{d u}{\xi(u)} .
$$


Д о к а з а т ел ь с т о. Выберем $\varepsilon \in(0,1)$. Из условия (4.2) следует, что при некоторых $\delta \in\left[0, t_{0}\right]$ и $\bar{x} \geqslant x_{0}$

$$
(1+\varepsilon)^{-1} \leqslant \frac{v(x+\delta \xi)}{v(x)} \leqslant 1+\varepsilon \quad \forall x \geqslant \bar{x} .
$$

Пусть $y \geqslant x \geqslant \bar{x}$. Положим $y_{0}=x$ и $y_{k+1}=y_{k}+\delta \xi_{k}, k \geqslant 0$, где $\xi_{k}=\xi\left(y_{k}\right)$. Последовательность $\left\{y_{k}\right\}$ монотонно возрастает к бесконечности. Действительно, из предположения $\lim y_{k}=c \in(x, \infty)$ следует, что $\lim \xi_{k}=\xi(c)=\lim \left(y_{k+1}-y_{k}\right) / \delta=0$, а это противоречит условию $\xi(x)>0$ для любого $x \geqslant \bar{x}$. Имеем по $(4.1)$

$$
1=\frac{y_{k+1}-y_{k}}{\delta \xi_{k}} \leqslant \frac{1}{\delta} \max _{y_{k} \leqslant u \leqslant y_{k+1}} \frac{\xi(u)}{\xi_{k}} \int_{y_{k}}^{y_{k+1}} \frac{d u}{\xi(u)} \leqslant k \int_{y_{k}}^{y_{k+1}} \frac{d u}{\xi(u)},
$$

где $k=\delta^{-1} \max _{0 \leqslant t \leqslant \delta} L(t)$. Отсюда при $n$ таком, что $y_{n} \leqslant y<y_{n+1}$, следует

$$
n \leqslant k \int_{y_{0}}^{y_{n}} \frac{d u}{\xi(u)} \leqslant k \int_{x}^{y} \frac{d u}{\xi(u)}
$$

Теперь

$$
\frac{v(y)}{v(x)}=\prod_{l=0}^{n-1} \frac{v\left(y_{l+1}\right)}{v\left(y_{l}\right)} \cdot \frac{v(y)}{v\left(y_{n}\right)}
$$

и, следовательно,

$$
(1+\varepsilon)^{-(1+n)} \leqslant \frac{v(y)}{v(x)} \leqslant(1+\varepsilon)^{1+n} .
$$

Отсюда и из оценки (4.4) вытекает утверждение леммы 3.

Сразу же отметим, что из условия (2.9) вытекает (4.1), а из леммы 3 - соотношение (2.14).

Далее докажем теорему 1В. Так же, как при доказательстве равенства (2.2), воспользуемся индукцией. Положим для краткости $p_{m}(y)=$ $p^{* m}(y), m \geqslant 1$. Пусть положительные $\eta$ и $\varepsilon$ достаточно медленно стремятся к бесконечности и нулю, соответственно, при $x \rightarrow \infty$ (от $\varepsilon=\varepsilon(x)$ требуется, чтобы $\varepsilon \xi(\beta x) \sqrt{g^{\prime \prime}(\beta x)} \rightarrow \infty$; по поводу выбора $\eta$ см. (4.23), (4.24) и ниже). Имеем, так же, как в (3.1),

$$
p_{m+1}(x)=I_{1}+I_{2}+I_{3} .
$$

В соответствии с $(2.10),(2.15)$ можно записать

$$
\begin{aligned}
& I_{2} \sim a_{m} \int_{\eta}^{x-\eta} u^{m}\left(\frac{y}{m}\right)\left(g^{\prime \prime}\left(\frac{y}{m}\right)\right)^{-(m-1) / 2} u(x-y) e^{-\tau(y)} d y=a_{m} \tilde{I}_{2}, \\
& x \rightarrow \infty, \text { где } a_{m}=(2 \pi)^{(m-1) / 2} / \sqrt{m}, \tau(y)=g(x-y)+m g(y / m) .
\end{aligned}
$$


Пусть $\alpha=m /(m+1), \beta=1-\alpha=1 /(m+1)$. Имеем

$$
\tau(\alpha x)=(m+1) g(\beta x), \quad \tau^{\prime}(\alpha x)=0, \quad \tau^{\prime \prime}(\alpha x)=\frac{1}{\alpha} g^{\prime \prime}(\beta x),
$$

$\tau^{\prime \prime}(y)>0, \quad y>\eta ; \quad \tau(y)=\tau(\alpha x)+\frac{1}{2}(y-\alpha x)^{2} \tau^{\prime \prime}(\theta), \quad \theta \in(y, \alpha x)$.

Далее,

$$
\tilde{I}_{2}=\int_{|y-\alpha x| \leqslant \varepsilon \xi(\beta x)} \cdots+\int_{\alpha x+\varepsilon \xi(\beta x)}^{x-\eta} \ldots+\int_{\eta}^{\alpha x-\varepsilon \xi(\beta x)} \cdots=I^{(1)}+I^{(2)}+I^{(3)},
$$

причем по (4.7) и (2.13)

$$
\begin{aligned}
I^{(1)} \sim & u^{m+1}(\beta x)\left(g^{\prime \prime}(\beta x)\right)^{(1-m) / 2} e^{-\tau(\alpha x)} \\
& \times \int_{|t| \leqslant \varepsilon \xi(\beta x)} \exp \left\{-\frac{1+o(1)}{2 \alpha} t^{2} g^{\prime \prime}(\beta x)\right\} d t \\
\sim & u^{m+1}(\beta x)\left(g^{\prime \prime}(\beta x)\right)^{-m / 2} e^{-\tau(\alpha x)} \sqrt{2 \pi \alpha}, \quad x \rightarrow \infty,
\end{aligned}
$$

еслии $\varepsilon \xi(\beta x) \sqrt{g^{\prime \prime}(\beta x)} \rightarrow \infty$.

Положим $v(t)=u^{m}(t)\left(g^{\prime \prime}(t)\right)^{-(m-1) / 2}$. С помощью леммы 3 получим при некотором положительном $k$

$$
\begin{array}{ll}
\frac{v(y / m)}{v(\beta x)}<2 e^{k(\omega(\beta x)-\omega(y / m))}, & \beta x \geqslant \frac{y}{m} \geqslant \frac{\eta}{m}, \\
\frac{v(x-y)}{v(\beta x)}<2 e^{k(\omega(x-y)-\omega(\beta x))}, & x-y \geqslant \beta x .
\end{array}
$$

Отсюда

$$
I^{(3)} \leqslant 4 u^{m+1}(\beta x)\left(g^{\prime \prime}(\beta x)\right)^{-(m-1) / 2} \int_{\eta}^{y_{0}} e^{-\nu(y)} d y,
$$

где $y_{0}=\alpha x-\varepsilon \xi(\beta x), \nu(y)=\tau(y)-k(\omega(x-y)-\omega(y / m))$. Положим $q(t)=k \xi^{\prime}(t)\left[\xi^{2}(t) g^{\prime \prime}(t)\right]^{-1}$. В силу условий $(2.9)$ и $(2.12)$ имеем

$$
\begin{aligned}
\nu^{\prime \prime}(y) & =g^{\prime \prime}(x-y)(1+q(x-y))+\frac{1}{m} g^{\prime \prime}\left(\frac{y}{m}\right)\left(1-\frac{1}{m} q\left(\frac{y}{m}\right)\right) \\
& =g^{\prime \prime}(x-y)(1+o(1))+\frac{1}{m} g^{\prime \prime}\left(\frac{y}{m}\right)(1+o(1)) \sim \tau^{\prime \prime}(y),
\end{aligned}
$$

$x, x-y \rightarrow \infty$. Следовательно, функция $\nu(y)$ выпукла и

$$
\nu(y) \geqslant \nu\left(y_{0}\right)+\left(y-y_{0}\right) \nu^{\prime}\left(y_{0}\right) \text {. }
$$

Далее,

$$
\begin{aligned}
\nu\left(y_{0}\right) & =\nu(\alpha x)+\left(y_{0}-\alpha x\right) \nu^{\prime}(\alpha x)+\frac{1}{2}\left(y_{0}-\alpha x\right)^{2} \nu^{\prime \prime}(\theta), \quad \theta \in\left(y_{0}, \alpha x\right), \\
\nu(\alpha x) & =\tau(\alpha x), \quad \nu^{\prime}(\alpha x)=\frac{k}{\alpha \xi(\beta x)},
\end{aligned}
$$


причем (см. (4.11), (2.12), (2.13) и (4.7))

$$
\nu^{\prime \prime}(\theta)=(1+o(1)) \tau^{\prime \prime}(\theta) \sim \tau^{\prime \prime}(\alpha x)=\frac{1}{\alpha} g^{\prime \prime}(\beta x), \quad x \rightarrow \infty .
$$

Отсюда

$$
\nu\left(y_{0}\right)=\tau(\alpha x)+\frac{1+o(1)}{2} \varepsilon^{2} \xi^{2}(\beta x) \tau^{\prime \prime}(\alpha x), \quad x \rightarrow \infty,
$$

и, аналогично,

$$
\nu^{\prime}\left(y_{0}\right) \sim \varepsilon \xi(\beta x) \tau^{\prime \prime}(\alpha x), \quad x \rightarrow \infty .
$$

Подставляя оценки (4.13) и (4.14) в (4.12), легко найдем

$$
\int_{\eta}^{y_{0}} e^{-\nu(y)} d y=e^{-\tau(\alpha x)} \frac{1}{\sqrt{g^{\prime \prime}(\beta x)}} o(1), \quad x \rightarrow \infty .
$$

Таким образом (см. (4.9), (4.10)), $I^{(3)}=o\left(I^{(1)}\right), x \rightarrow \infty$. Аналогичная оценка справедлива и для $I^{(2)}$. Поэтому $I^{(2)}+I^{(3)}=o\left(I^{(1)}\right), x \rightarrow \infty$ и, следовательно (см. (4.6), (4.8) и (4.9)),

$$
I_{2} \sim a_{m+1}\left(g^{\prime \prime}\left(\frac{x}{m+1}\right)\right)^{-m / 2} p^{m+1}\left(\frac{x}{m+1}\right), \quad x \rightarrow \infty .
$$

Теперь оценим $I_{1}$ и $I_{3}$ (см. (4.5)). Очевидно,

$$
I_{3} \leqslant \sup _{y \geqslant x-\eta} p_{m}(y), \quad I_{1} \leqslant \sup _{y \geqslant x-\eta} p(y) .
$$

Далее приведем один вспомогательный результат.

Лемма 4. Пусть $v(x)$ удовлетворяет условию

$$
\liminf _{x \rightarrow \infty} x^{2} v^{\prime \prime}(x)>0 \text {. }
$$

Тогда при $x \uparrow \infty$

$$
\frac{v(x)}{x} \uparrow \lambda \leqslant \infty, \quad v^{\prime}(x) \uparrow \lambda, \quad \kappa(x)=x v^{\prime}(x)-v(x) \uparrow \infty, \quad \frac{\kappa(x)}{v^{\prime}(x)} \rightarrow \infty .
$$

Д ок аза те л ь с т в о. Очевидно, $\kappa^{\prime}(x)=x v^{\prime \prime}(x)>0$, начиная с некоторого места. Поэтому $\kappa(x) \uparrow c \leqslant \infty$. Предположение $c<\infty$ противоречит соотношению $x^{2} v^{\prime \prime}(x) \geqslant \delta>0$, поскольку при $x \rightarrow \infty$

$$
\delta \leqslant \int_{x}^{e x} \frac{x^{2} v^{\prime \prime}(x)}{x} d x=\int_{x}^{e x} \kappa^{\prime}(x) d x=\kappa(e x)-\kappa(x) \longrightarrow c-c=0 .
$$

Таким образом, $\kappa(x) \uparrow \infty$. Далее, $(v(x) / x)^{\prime}=\kappa(x) / x^{2}>0$, откуда $v(x) / x \uparrow \lambda \leqslant \infty$, и, следовательно,

$$
\lim _{x \rightarrow \infty} \frac{v(x)}{x}=\lambda
$$


Имеем $v^{\prime}(x) \uparrow \bar{\lambda}$, и поэтому $v^{\prime}(y) \leqslant(v(x)-v(y)) /(x-y) \leqslant v^{\prime}(x)$ при любых достаточно больших $x>y$. Отсюда и из (4.17) несложно заключить, что $\bar{\lambda}=\lambda$.

Последнее утверждение леммы 4 проверяется с помощью правила Лопиталя при $\lambda=\infty$ и очевидно в противном случае. Лемма 4 доказана.

Вернемся к соотношению (4.16). Вначале покажем, что

$$
\sup _{y \geqslant x-\eta} p_{m}(y) \leqslant c_{1} p_{m}(x-\eta)
$$

где $c_{1}$ не зависит от $x$.

В самом деле, по индукционному предположению, в силу (2.10), (2.15), (2.13) и по лемме 3

$$
\begin{aligned}
\frac{p_{m}(y)}{p_{m}(x-\eta)} \leqslant & \left(\frac{g^{\prime \prime}(y / m)}{g^{\prime \prime}((x-\eta) / m)}\right)^{(m-1) / 2}\left(\frac{u(y / m)}{u((x-\eta) / m)}\right)^{m} \\
& \times \exp \left\{-m\left(g\left(\frac{y}{m}\right)-g\left(\frac{x-\eta}{m}\right)\right)\right\} \\
\leqslant & c_{1} \exp \left\{-m \int_{(x-\eta) / m}^{y / m} \tilde{g}^{\prime}(t) d t\right\}
\end{aligned}
$$

где

$$
\tilde{g}(t)=g(t)-k \omega(t)
$$

$k$ - некоторая положительная постоянная.

Докажем, что при условиях (2.12), (2.13)

$$
\xi g^{\prime}(x) \rightarrow \infty, \quad x \rightarrow \infty .
$$

Пусть $\left|\xi^{\prime}(x)\right| \leqslant L$, где $L>1$. Положим $\delta=(2 L)^{-1}$. Функция $g^{\prime}(x)$ монотонно возрастает. Поэтому

$$
\begin{aligned}
g^{\prime}(x) & \geqslant g^{\prime}(x)-g^{\prime}(x-\delta \xi)=\delta \xi g^{\prime \prime}(x-\theta \xi) \\
& =\delta \xi g^{\prime \prime}(x) \frac{g^{\prime \prime}(x-\theta \xi)}{g^{\prime \prime}(x)}, \quad 0<\theta<\delta .
\end{aligned}
$$

По $(2.13)$ и лемме $3 g^{\prime \prime}(x-\theta \xi) / g^{\prime \prime}(x) \geqslant e^{-1-k(\omega(x)-\omega(x-\delta \xi))}$, но

$$
\begin{aligned}
\omega(x)-\omega(x-\delta \xi) & \leqslant \delta \frac{\xi}{\xi\left(x-\theta_{1} \xi\right)}=\frac{\delta \xi}{\xi-\left(\xi-\xi\left(x-\theta_{1} \xi\right)\right)} \\
& \leqslant \frac{\delta \xi}{\xi-\delta \xi L}=\frac{\delta}{1-\delta L}=\frac{1}{L} .
\end{aligned}
$$

Таким образом,

$$
g^{\prime}(x) \geqslant \frac{1}{2 e L} e^{-k / L} \xi g^{\prime \prime}(x)=\delta_{0} \xi g^{\prime \prime}(x) .
$$

Соотношение (4.21) вытекает из (4.22) и (2.12).

Из (4.21) следует, что $\widetilde{g}^{\prime}(t) \geqslant 0$ при всех достаточно больших $t$. Отсюда и из (4.19) получим (4.18). 
Теперь покажем, что функцию $\eta=\eta(x) \rightarrow \infty$ можно подобрать таким образом, чтобы (см. (4.15))

$$
p_{m}(x-\eta)=o\left(I_{2}\right), \quad x \rightarrow \infty .
$$

Рассуждая, как в (4.19), найдем, что соотношение (4.23) следует из условия $\exp \left\{(m+1) g\left(\frac{x}{m+1}\right)-m g\left(\frac{x-\eta}{m}\right)+k \omega\left(\frac{x-\eta}{m}\right)\right\} \rightarrow 0, \quad x \rightarrow \infty$ которое, в свою очередь, вытекает из неравенства

$$
m \tilde{g}\left(\frac{x-\eta}{m}\right)-(m+1) \tilde{g}(\beta x) \geqslant \bar{k} \omega(\beta x) .
$$

Здесь $\widetilde{g}$ определено в $(4.20), \beta=(m+1)^{-1}, k, \bar{k}$ - некоторые положительные постоянные.

Левую часть (4.24) представим в виде $\widetilde{\kappa}(\beta x)-\eta \widetilde{g}^{\prime}(\beta x)$, где $\widetilde{\kappa}(u)=$ $u \widetilde{g}^{\prime}(u)-\widetilde{g}(u)$.

Из леммы 4 при $v(x)=\widetilde{g}(x)$ следует, что $\tau(x)=\widetilde{\kappa}(\beta x) / \widetilde{g}^{\prime}(\beta x) \rightarrow \infty$, $x \rightarrow \infty$.

Пусть $\eta=\frac{1}{2} \min (\beta x, \tau(x))$. Тогда $\widetilde{\kappa}(\beta x)-\eta \widetilde{g}^{\prime}(\beta x) \geqslant \frac{1}{2} \widetilde{\kappa}(\beta x)$.

Чтобы доказать (4.24), а следовательно, и (4.23), достаточно убедиться в том, что $\widetilde{\kappa}(\beta x) / \omega(\beta x) \rightarrow \infty, x \rightarrow \infty$. Действительно, $\widetilde{\kappa}(\beta x) \uparrow \infty$ по лемме 4. Применяя правило Лопиталя, видим

$$
\lim _{u \rightarrow \infty} \frac{\widetilde{\kappa}(u)}{\omega(u)}=\lim _{u \rightarrow \infty} \frac{\widetilde{\kappa}^{\prime}(u)}{\omega^{\prime}(u)}=\lim _{u \rightarrow \infty} u \widetilde{g}^{\prime \prime}(u) \xi(u)
$$

но (см. (4.20))

$$
u \widetilde{g}^{\prime \prime}(u) \xi(u)=u \xi(u) g^{\prime \prime}(u)\left(1+\frac{k \xi^{\prime}(u)}{\xi^{2}(u) g^{\prime \prime}(u)}\right) \sim u \xi(u) g^{\prime \prime}(u) \rightarrow \infty,
$$

при $u \rightarrow \infty$, по условиям (2.9), (2.12).

Из $(4.18),(4.23)$ и (4.16) следует, что $I_{3}=o\left(I_{2}\right), x \rightarrow \infty$. Соотношение $I_{1}=o\left(I_{2}\right), x \rightarrow \infty$, проверяется аналогично. Индукционный переход вытекает из сказанного и (4.5), (4.15). Теорема 1В доказана.

Перейдем к доказательству теоремы $2 \mathrm{~B}$, которое проведем аналогично доказательству теоремы 2 A. Прежде всего покажем, что в условиях теоремы $2 \mathrm{~B}$ имеет место (3.5). Для этого нам понадобятся аналоги соотношений (3.6). Найдем их.

Положим $\tau(y)=g(y)-h y, h<\lambda$, и пусть $y_{*}=y_{*}(h)$ является решением уравнения

$$
\tau^{\prime}\left(y_{*}\right)=0
$$


(в соответствии с одним из утверждений леммы 4 при $v(y)=g(y)$ такое решение существует и единственно при любом $h$, достаточно близком к $\lambda$ ). Заметим, что $y_{*} \uparrow \infty$ при $h \uparrow \lambda$; кроме того (см. (2.12)),

$$
\begin{aligned}
& \tau(y)=\tau\left(y_{*}\right)+\frac{1}{2}\left(y-y_{*}\right)^{2} g^{\prime \prime}(\theta), \quad \theta \in\left(y, y_{*}\right), \\
& \tau(y) \geqslant \tau\left(y_{0}\right)+\left(y-y_{0}\right) \tau^{\prime}\left(y_{0}\right), \quad y, y_{0} \geqslant x_{0} .
\end{aligned}
$$

Имеем

$$
\begin{aligned}
L(h) & =\left(\int_{-\infty}^{\eta}+\int_{\eta}^{y_{*}-\varepsilon \xi_{*}}+\int_{y_{*}-\varepsilon \xi_{*}}^{y_{*}+\varepsilon \xi_{*}}+\int_{y_{*}+\varepsilon \xi_{*}}^{\infty}\right) e^{h y} p(y) d y \\
& =I_{1}+\cdots+I_{4},
\end{aligned}
$$

где $\xi_{*}=\xi\left(y_{*}\right)$ (см. (2.9)) и, здесь и в дальнейшем предполагается, что $\eta \rightarrow \infty$ достаточно медленно, а положительное $\varepsilon \rightarrow 0$ при $h \uparrow \lambda$ таким образом, что $\varepsilon y_{*} \sqrt{g^{\prime \prime}\left(y_{*}\right)} \rightarrow \infty$.

Очевидно,

$$
I_{1} \leqslant e^{h \eta}
$$

Далее, так же, как в (4.6), (4.8), (4.9), найдем

$$
I_{3} \sim e^{-\tau\left(y_{*}\right)} u\left(y_{*}\right) \sqrt{\frac{2 \pi}{g^{\prime \prime}\left(y_{*}\right)}}, \quad h \uparrow \lambda .
$$

Оценим $I_{4}$ и $I_{2}$. Предварительно отметим (см. (4.25), (4.26)), что

$$
\begin{aligned}
\tau\left(y_{*} \pm \varepsilon \xi_{*}\right) & =\tau\left(y_{*}\right)+\frac{1}{2} \varepsilon^{2} \xi_{*}^{2} g^{\prime \prime}\left(\theta_{1}\right), \\
\tau^{\prime}\left(y_{*} \pm \varepsilon \xi_{*}\right) & = \pm \varepsilon \xi_{*} g^{\prime \prime}\left(\theta_{2}\right), \quad \theta_{1}, \theta_{2} \in\left(y_{*}-\varepsilon \xi_{*}, y_{*}+\varepsilon \xi_{*}\right) .
\end{aligned}
$$

Положим $y_{0}=y_{*}+\varepsilon \xi_{*}, \nu(y)=\tau(y)-k \int_{y_{*}}^{y} d u / \xi(u)\left(y>y_{*}\right)$, где $k>0$ достаточно большая постоянная. По $(4.26),(2.10)$ и лемме 3 имеем

$$
I_{4} \leqslant c u\left(y_{*}\right) \int_{y_{0}}^{\infty} e^{-\nu(y)} d y,
$$

причем по $(2.9),(2.12)$ и $(2.13)$

$$
\begin{aligned}
\nu\left(y_{0}\right) & =\tau\left(y_{*}\right)-\varepsilon k+\frac{1}{2} \varepsilon^{2} \xi_{*}^{2} \nu^{\prime \prime}\left(\theta_{1}\right), \\
\nu^{\prime}\left(y_{0}\right) & =-\frac{k}{\xi_{*}}+\varepsilon \xi_{*} \nu^{\prime \prime}\left(\theta_{2}\right), \quad \theta_{1}, \theta_{2} \in\left(y_{*}, y_{0}\right), \\
\nu^{\prime \prime}\left(\theta_{i}\right) & \sim g^{\prime \prime}\left(\theta_{i}\right) \sim g^{\prime \prime}\left(y_{*}\right), \quad h \uparrow \lambda .
\end{aligned}
$$

Кроме того, справедлива оценка (4.12).

Из сказанного следует, что

$$
\begin{aligned}
\int_{y_{0}}^{\infty} e^{-\nu(y)} d y & =O(1) e^{-\tau\left(y_{*}\right)-\varepsilon^{2} \xi_{*}^{2} g^{\prime \prime}\left(y_{*}\right) / 3} \int_{0}^{\infty} e^{-t \varepsilon \xi_{*} g^{\prime \prime}\left(y_{*}\right) / 2} d t \\
& =O(1) \frac{e^{-\tau\left(y_{*}\right)}}{\sqrt{g^{\prime \prime}\left(y_{*}\right)}} \frac{e^{-\varepsilon^{2} \xi_{*}^{2} g^{\prime \prime}\left(y_{*}\right) / 3}}{\varepsilon \xi_{*} \sqrt{g^{\prime \prime}\left(y_{*}\right)}}=o(1) \frac{e^{-\tau\left(y_{*}\right)}}{\sqrt{g^{\prime \prime}\left(y_{*}\right)}}, \quad h \uparrow \lambda,
\end{aligned}
$$


откуда (см. (4.31), (4.29))

$$
I_{4}=o\left(I_{3}\right), \quad h \uparrow \lambda .
$$

Аналогично проверяется, что

$$
I_{2} \leqslant c u\left(y_{*}\right) \int_{\eta}^{y_{*}-\varepsilon \xi_{*}} \exp \left\{-\tau(y)+k \int_{y}^{y_{*}} \frac{d u}{\xi(u)}\right\} d y=o\left(I_{3}\right), \quad h \uparrow \lambda .
$$

Наконец, покажем, что

$$
I_{1}=o\left(I_{3}\right), \quad h \uparrow \lambda .
$$

Из (4.29) и (2.14) следует, что равенство (4.34) является следствием соотношения $-\tau\left(y_{*}\right)-k \omega\left(y_{*}\right)-h \eta \rightarrow \infty, h \uparrow \lambda$. Учитывая, что $h=g^{\prime}\left(y_{*}\right)$ по (4.25), $\mathrm{a}-\tau\left(y_{*}\right)=\kappa\left(y_{*}\right)=y_{*} g^{\prime}\left(y_{*}\right)-g\left(y_{*}\right)$, имеем

$$
-\tau\left(y_{*}\right)-k \omega\left(y_{*}\right)-h \eta=\kappa\left(y_{*}\right)\left(1-\frac{k \omega\left(y_{*}\right)}{\kappa\left(y_{*}\right)}-\eta \frac{g^{\prime}\left(y_{*}\right)}{\kappa\left(y_{*}\right)}\right) \sim \kappa\left(y_{*}\right) \rightarrow \infty,
$$

при $y_{*} \rightarrow \infty$, где последнее утверждение имеет место, поскольку $\kappa(y) / \omega(y) \rightarrow \infty$ и $\kappa(y) / g^{\prime}(y) \rightarrow \infty$, если $y \rightarrow \infty$ (см. лемму 4 при $v=g$ и рассуждения, использованные в процессе доказательства оценки (4.23)). Соотношение (4.34), таким образом, установлено.

Из (4.32)-(4.34), (4.29) и (4.27) следует, что

$$
L(h) \sim e^{-\tau\left(y_{*}\right)} u\left(y_{*}\right) \sqrt{\frac{2 \pi}{g^{\prime \prime}\left(y_{*}\right)}}, \quad h \uparrow \lambda .
$$

Теперь исследуем асимптотическое поведение $m(h), \sigma^{2}(h)$ и $\alpha_{k}(h)$ (см. (3.9) и выше). Вначале покажем, что при $k=1,2, \ldots$

$$
\begin{aligned}
J_{k} & =\int_{-\infty}^{\infty}\left(y-y_{*}\right)^{k} e^{h y} p(y) d y \\
& =\left(\alpha_{k}+o(1)\right)\left(g^{\prime \prime}\left(y_{*}\right)\right)^{-k / 2} L(h), \quad h \uparrow \lambda,
\end{aligned}
$$

где $\alpha_{k}-k$-й момент стандартного нормального распределения.

Действительно, $J_{k}=I_{1}+\cdots+I_{4}$, причем интегралы $I_{i}, i=1, \ldots, 4$, имеют такие же пределы, как в (4.27), и оцениваются аналогично. Так, при $h \uparrow \lambda$ имеем: $\left|I_{1}\right|=O\left(y_{*}^{k} e^{h \eta}\right)$,

$$
\begin{aligned}
I_{3} & \sim u\left(y_{*}\right) e^{-\tau\left(y_{*}\right)}\left(g^{\prime \prime}\left(y_{*}\right)\right)^{-k} \int_{|t| \leqslant \varepsilon \xi_{*} \sqrt{g^{\prime \prime}\left(y_{*}\right)}} t^{k} e^{-\left(1+o(1) t^{2} / 2\right.} d t \\
& =\left(\alpha_{k}+o(1)\right)\left(g^{\prime \prime}\left(y_{*}\right)\right)^{-k / 2} L(h), \\
I_{2}+I_{4} & =o(1)\left(g^{\prime \prime}\left(y_{*}\right)\right)^{-k / 2} L(h) .
\end{aligned}
$$

Отсюда следует (4.36). Имеем

$$
m(h)=y_{*}+\frac{1}{L(h)} J_{1}
$$


и, следовательно,

$$
m(h)=y_{*}\left(1+o\left(\frac{1}{z}\right)\right), \quad h \uparrow \lambda,
$$

здесь и далее $z=y_{*} \sqrt{g^{\prime \prime}\left(y_{*}\right)}$ (напоминаем об условиях $(2.9)$ и $(2.12)$ ).

Из (4.38), в частности, следует, что

$$
\begin{aligned}
\sigma^{2}(h) & =\mathbf{D} X^{(h)}=\mathbf{E}\left(X^{(h)}-y_{*}\right)^{2}-\left(m(h)-y_{*}\right)^{2} \\
& =\frac{1}{L(h)} J_{2}+o(1)\left(\frac{y_{*}}{z}\right)^{2} \sim\left(\frac{y_{*}}{z}\right)^{2}, \quad h \uparrow \lambda .
\end{aligned}
$$

Похожие рассуждения приводят к оценке

$$
\left(\sigma^{2}(h)\right)^{\prime}=\mathbf{E}\left(X^{(h)}-m(h)\right)^{3}=o\left(\frac{y_{*}}{z}\right)^{3}, \quad h \uparrow \lambda,
$$

и к более общим соотношениям

$$
\mathbf{E}\left(X^{(h)}-m(h)\right)^{k}=\left(\alpha_{k}+o(1)\right)\left(\frac{y_{*}}{z}\right)^{k}, \quad h \uparrow \lambda .
$$

Заметим, что из (4.39), (4.41) (и (2.10)) вытекает условие (3.11).

Докажем теперь, что при условиях (2.9)-(2.13), (1.3) имеет место (3.7). Так же, как при доказательстве теоремы $2 \mathrm{~A}$, ограничимся случаем $\varepsilon<\lambda_{0}<h<\lambda$, выбирая постоянную $\lambda_{0}$ достаточно близкой к $\lambda$.

Пусть положительная постоянная $x_{0}$ достаточно велика. Имеем по (4.35), (4.39)

$$
\begin{aligned}
\sigma(h) \sup _{x \leqslant x_{0}} p_{h}^{* m}(x) & \leqslant \frac{\sigma(h)}{L^{m}(h)} e^{h x_{0}} \leqslant c u^{-m}\left(y_{*}\right)\left(g^{\prime \prime}\left(y_{*}\right)\right)^{(m-1) / 2} e^{m \tau\left(y_{*}\right)+h x_{0}} \\
& \leqslant c_{1}, \quad \lambda_{0} \leqslant h<\lambda
\end{aligned}
$$

где последняя оценка вытекает из того, что (см. (4.34) и ниже) $\tau\left(y_{*}\right)+$ $k \omega\left(y_{*}\right)+h x_{0} \rightarrow-\infty$ при $y_{*} \rightarrow \infty$. Далее, по $(2.15),(4.35),(4.39),(4.25)$ и лемме 3

$$
\begin{aligned}
\sigma(h) \sup _{x>x_{0}} p_{h}^{* m}(x) & \leqslant c \frac{\sigma(h)}{L^{m}(h)} \sup _{x>x_{0}} e^{h x-m g(x / m)} u^{m}\left(\frac{x}{m}\right)\left(g^{\prime \prime}\left(\frac{x}{m}\right)\right)^{(1-m) / 2} \\
& \leqslant c_{1} \sup _{x>x_{0}}\left(\frac{u(x / m)}{u\left(y_{*}\right)}\right)^{m}\left(\frac{g^{\prime \prime}(x / m)}{g^{\prime \prime}\left(y_{*}\right)}\right)^{(1-m) / 2} e^{-m\left(\tau(x / m)-\tau\left(y_{*}\right)\right)} \\
& \leqslant c_{1} \sup _{y>\bar{y}} e^{-m\left(\nu(x / m)-\nu\left(y_{*}\right)\right)}
\end{aligned}
$$

где $\bar{y}=x_{0} / m, \nu(y)=\tau(y)-k\left|\int_{y_{*}}^{y} d u / \xi(u)\right|$.

Если $\left|y-y_{*}\right| \leqslant \xi_{*}=\xi\left(y_{*}\right)$, то (см. (4.30), (4.31) и ниже) $\nu(y)-\nu\left(y_{*}\right) \geqslant$ $-\left|y-y_{*}\right| k / \xi_{*} \geqslant-k$; если же $y>y_{0}=y_{*}+\xi_{*}$, то

$\nu(y)-\nu\left(y_{*}\right) \geqslant \nu\left(y_{0}\right)-\nu\left(y_{*}\right)+\left(y-y_{0}\right) \nu^{\prime}\left(y_{0}\right) \geqslant-k+\left(-\frac{k}{\xi_{*}}+\xi_{*} \nu^{\prime \prime}\left(\theta_{2}\right)\right) \geqslant-k$.

Такая же оценка справедлива и для $\bar{y} \leqslant y<y_{*}-\xi_{*}$. 
Из сказанного, (4.43) и (4.42) следует оценка (3.7).

Доказательство теоремы 2В вытекает из (3.4), (3.5), (3.8)-(3.13).

Доказательство следствия 1В проводится в целом так же, как доказательство следствия $1 \mathrm{~A}$. В некоторых комментариях нуждается лишь условие (3.20) при $u \in\left(x, x+y_{n}\right)$ и $h \geqslant \lambda_{0}$ (или $x \geqslant n m\left(\lambda_{0}\right)$ ), где постоянная $\lambda_{0}$ достаточно близка к $\lambda$ (см. (1.5) и (2.11)).

Чтобы убедиться в справедливости соотношения (3.20) в условиях теоремы 2В, требуется доказать, что при всех достаточно больших $x$ и всех $h=h(u / n) \geqslant \lambda_{0}$

$$
\frac{1}{2}<\frac{\sigma(h)}{\sigma(\widehat{h})}<2, \quad u \in\left(x, x+y_{n}\right) .
$$

Итак, проверим (4.44). Из (4.25), (3.15) (и (1.5)) следует, что $\sigma^{-2}(h)=$ $h_{u}^{\prime}=g^{\prime \prime}\left(y_{*}\right)\left(y_{*}\right)_{u}^{\prime}$, т.е. $\left(y_{*}\right)_{u}^{\prime}=\left(n \sigma^{2}(h) g^{\prime \prime}\left(y_{*}\right)\right)^{-1}$. Учитывая (4.39), отсюда получим

$$
\left(y_{*}\right)_{u}^{\prime} \sim \frac{1}{n}, \quad h \uparrow \lambda .
$$

Пусть $\widehat{y}_{*}=y_{*}(\widehat{h}), \bar{y}_{*}=y_{*}(\bar{h})$, где, как в $(3.17),(3.26), \widehat{h}=h(x / n), \bar{h}=$ $h\left(\left(x+y_{n}\right) / n\right), y_{n}=\sqrt{n} \sigma(\widehat{h})$. Имеем при $u \in\left(x, x+y_{n}\right)$ и $x \geqslant n m\left(\lambda_{0}\right)$

$$
y_{*}-\widehat{y}_{*}=\left.u\left(y_{*}\right)_{u}^{\prime}\right|_{u=\tilde{u}}, \quad \tilde{u} \in\left(x, x+y_{n}\right),
$$

откуда по (4.45)

$$
\widehat{y}_{*} \leqslant y_{*} \leqslant \widehat{y}_{*}+(1+o(1)) \frac{y_{n}}{n}, \quad h \uparrow \lambda .
$$

По (4.39)

$$
\frac{y_{n}}{n}=\frac{1}{\sqrt{n}} \sigma(\widehat{h}) \sim \frac{1}{\sqrt{n}} \frac{1}{\sqrt{\xi_{*}^{2} g^{\prime \prime}\left(y_{*}\right)}} \xi_{*}, \quad h \uparrow \lambda,
$$

где $\xi_{*}=\xi\left(y_{*}\right)$.

Поскольку $h \in(\widehat{h}, \bar{h})$ при $u \in\left(x, x+y_{n}\right)$, имеем с учетом (4.39), (4.46), $(4.47),(2.12)$ и леммы 3 (см. также рассуждение перед оценкой (4.22))

$$
\sigma^{2}(h) \sim \frac{1}{g^{\prime \prime}\left(y_{*}\right)} \sim \frac{1}{\widehat{g}^{\prime \prime}\left(y_{*}\right)} \sim \sigma^{2}(\widehat{h}), \quad h \uparrow \lambda .
$$

Соотношение (4.44) вытекает отсюда при всех достаточно больших $n$, если $\lambda_{0}$ близко к $\lambda$. Остальные рассуждения, доказывающие следствие 1В (см. (3.20)-(3.26)), не меняются. Заметим только, что по (4.25), $(4.22)$ и $(2.12)$

$$
h \sigma(h) \sim \frac{g^{\prime}\left(y_{*}\right)}{\sqrt{g^{\prime \prime}\left(y_{*}\right)}} \longrightarrow \infty, \quad h \uparrow \lambda,
$$

и, следовательно, $\inf _{x \geqslant n m\left(\lambda_{0}\right)} \hat{h} \sigma(\widehat{h})>0$.

Следствие $1 \mathrm{~B}$ доказано. 
Проверим следствие 2В. Равенство (2.18) вытекает непосредственно из теоремы 2B, а соотношение $(2.17)$ - из $(2.18),(4.35),(4.39),(2.13)$ и того, что при $h=h(t)$

$$
\tau(t)-\tau\left(y_{*}\right)=\left(t-y_{*}\right)^{2} g^{\prime \prime}(\theta), \quad \theta \in\left(t, y_{*}\right),
$$

и (см. (4.38))

$$
t-y_{*}=o\left(\frac{1}{\sqrt{g^{\prime \prime}\left(y_{*}\right)}}\right)=o\left(\xi\left(y_{*}\right)\right), \quad h \uparrow \lambda .
$$

Заметим также (см. (2.14) и (4.21)), что при $t \rightarrow \infty$

$$
-\ln p(t)+O\left(1+\left|\ln g^{\prime \prime}(t)\right|\right)=g(t)+O(\omega(t)), \quad \text { a } \omega(t)=o(g(t)) .
$$

5. Доказательства теорем 1C-2D. Примеры. Доказательства теорем $1 \mathrm{C}$ и $2 \mathrm{C}$ идентичны доказательствам теорем $1 \mathrm{~A}$ и $2 \mathrm{~A}$ соответственно. Отметим лишь, что при условии (2.19)

$$
p^{*(m+1)}(x)=\int_{x}^{0} p(x-y) p^{* m}(y) d y, \quad x<0,
$$

и, кроме того, имеют место соотношения (3.6), в которых $\delta=h \rightarrow \infty$, и справедливы условия (2.3) и (3.11) (см. [2, теорема 7.3]). Условие (3.7) также выполняется. Так,

$$
\begin{aligned}
& \sigma(h) \sup _{x_{0}<x<0} p_{h}^{* m}(x) \leqslant c(m) \frac{\sigma(h)}{L^{m}(h)} \sup _{x_{0}<x<0} e^{h x} x^{m \rho-1} l^{m}(x) \\
& \quad \leqslant \frac{3 \sqrt{\rho}}{\Gamma(m \rho)} \sup _{0<t<h\left|x_{0}\right|} e^{-t} t^{m \rho-1}\left(\frac{l(t / h)}{l(1 / h)}\right)^{m}=O(1), \quad h \rightarrow \infty .
\end{aligned}
$$

Вывод из теоремы $2 \mathrm{C}$ следствия $1 \mathrm{C}$ лишь несущественными деталями отличается от рассуждений (3.14)-(3.26). В частности, сохраняются соотношения (3.19) (при $\delta=h$ и $\lambda=\infty$ ) и (3.20).

Теорема 1D доказывается аналогично теореме 1B с некоторыми упрощениями, возникающими благодаря равенству (5.1). Так, при условиях теоремы $1 \mathrm{D}$ и в обозначениях из (4.5)-(4.7)

$$
\begin{aligned}
p_{m+1}(x) \sim a_{m}( & \left.\int_{|y-\alpha x| \leqslant \varepsilon \xi(\beta x)}+\int_{\alpha x+\varepsilon \xi(\beta x)}^{0}+\int_{x}^{\alpha x-\varepsilon \xi(\beta x)}\right) \\
& u^{m}\left(\frac{y}{m}\right)\left(g^{\prime \prime}\left(\frac{y}{m}\right)\right)^{-(m-1) / 2} u(x-y) e^{-\tau(y)} d y,
\end{aligned}
$$

$x \rightarrow-0$. Здесь интегралы оцениваются точно так же, как интегралы $I^{(1)}, I^{(2)}$ и $I^{(3)}$ из (4.8). При этом используются соответствующие модификации леммы 3 и соотношений (4.10)-(4.15). Заметим, что оценивать величины $I_{1}$ и $I_{3}$ (см. (4.5) и (4.6)) при доказательстве теоремы $1 \mathrm{D}$ нет необходимости! 
Доказательство теоремы 2D также вполне аналогично доказательству теоремы 2В. Оценивая, например, $L(h)$, можно воспользоваться равенством (4.27), положив в нем $\eta=-\sqrt{\tau\left(y_{*}\right) / g^{\prime}\left(y_{*}\right)}$ и заменив верхний предел в $I_{4}$ нулем. Оценивание интегралов $I_{2}, I_{3}$ и $I_{4}$ производится так же, как в (4.29)-(4.33). Аналог соотношения (4.34) тоже справедлив. Действительно, рассуждая, как в лемме 4, найдем, что при условии $(2.23) g^{\prime}(y) \uparrow \infty$ и $\kappa(y)=g(y)-y g^{\prime}(y) \uparrow \infty$, если $y \uparrow-0$. Отсюда следует, что

$$
\lim _{y \uparrow-0} \frac{g^{\prime}(y)}{\kappa(y)}=\lim _{y \uparrow-0} \frac{g^{\prime \prime}(y)}{\kappa^{\prime}(y)}=\infty \quad \text { и } \quad \lim _{y \uparrow-0} \frac{\kappa(y)}{\omega(y)}=\lim _{y \uparrow-0} \frac{\kappa^{\prime}(y)}{\omega^{\prime}(y)}=\infty .
$$

Остальные рассуждения вовсе не меняются или проводятся с незначительными модификациями.

К п р и м е р у А. Если $p(x)=e^{x} /\left(1+e^{x}\right)^{2}$, то (см. (1.1) и [12])

$$
L(h)=\frac{\pi h}{\sin \pi h}, \quad 0<h<1 .
$$

Пусть $\delta=1-h$. Тогда $L(h)=((1-\delta) / \delta) L(\delta)$ и $($ см. [13])

$$
\ln L(\delta)=\sum_{m \geqslant 1} \frac{c_{m}}{(2 m) !} \delta^{2 m}
$$

где $c_{m}=\left((2 \pi)^{2 m} /(2 m)\right) B_{m}$, а $\left\{B_{m}\right\}$ - числа Бернулли $\left(B_{1}=\frac{1}{6}\right.$, $\left.B_{2}=\frac{1}{30}, \ldots\right)$.

Из (1.2), (5.3) и (5.4) следует, что уравнение (1.5) при достаточно малых положительных $\delta$ и $\nu=n / x$ равносильно соотношению

$$
\frac{1}{1-\delta}+\frac{1}{\delta}-(\ln L(\delta))_{\delta}^{\prime}=\frac{1}{\delta}+\sum_{k \geqslant 0} \delta^{k}-\sum_{m \geqslant 1} \frac{c_{m}}{(2 m-1) !} \delta^{2 m-1}=\frac{\psi(\delta)}{\delta}=\nu^{-1}
$$

или $\delta / \psi(\delta)=\nu$. Применяя формулу Лагранжа [13], отсюда получим

$$
\delta=\sum_{k \geqslant 1} \frac{\alpha_{k}}{k !} \nu^{k}
$$

где $\alpha_{k}=\left.\left(\psi^{k}(t)\right)^{(k-1)}\right|_{t=0}$. В частности, $\alpha_{1}=1, \alpha_{2}=2, \alpha_{3}=12-2 \pi^{2}$.

Пусть

$$
s(\nu)=\ln L(h)-\frac{h}{\nu}+\frac{1}{\nu^{2}}+\ln \nu
$$

где $h$ - решение уравнения (1.5), или (см. (5.5)) $h=1-\delta=1-$ $\sum_{k \geqslant 1} \alpha_{k} \nu^{k} / k$ !. Тогда (см. (3.15))

$$
s^{\prime}(\nu)=-\frac{1}{\nu}-\frac{\delta}{\nu^{2}}=-\sum_{k \geqslant 2} \frac{\alpha_{k}}{k !} \nu^{k-2}
$$


откуда, принимая во внимание, что $s(0)=0$, найдем

$$
s(\nu)=1-\sum_{k \geqslant 1} \frac{\gamma_{k}}{k !} \nu^{k}, \quad \gamma_{k}=\frac{\alpha_{k+1}}{k(k+1)} .
$$

Учитывая $(5.3),(5.5)$ и то, что $\sigma^{2}(h)=(\ln L(\delta))^{\prime \prime}$, получим

$$
\frac{1}{\sigma(h)}=\frac{\delta}{\nu}\left(\delta^{2} \sigma^{2}(h)\right)^{-1 / 2}=1+v(\nu),
$$

где функция $v$ представима степенным рядом.

Рассуждая аналогично, можно показать (см. замечание 2), что

$$
\lambda_{m+2}(h)=(m+1) !\left(1-\frac{m+2}{6}\left(\pi^{2}-3\right) \nu^{2}+\cdots\right) .
$$

Утверждение примера А следует из (5.3)-(5.9), (2.8) и (2.5).

К п р и м е р у В. Здесь (см. [12]) $L(h)=\Gamma(1+h)$ и (см. [13]) при $h \rightarrow \infty$

$$
m(h) \sim \ln h+\frac{1}{2 h}-\sum_{k \geqslant 1} \frac{B_{k}}{2 k} h^{-2 k}=\ln h+\phi\left(\frac{1}{h}\right) .
$$

Положим $t=x / n, \nu=e^{-t}$. Тогда с учетом (5.10) уравнение (1.5) можно записать в виде $h^{-1} \phi(1 / h)=\nu$, откуда по формуле Лагранжа

$$
\begin{aligned}
& \left.\frac{1}{h} \sim \sum_{k \geqslant 1} \frac{\nu^{k}}{k !}\left(e^{k \phi(t)}\right)^{(k-1)}\right|_{t=0}=\nu+\frac{1}{2} \nu^{2}+\frac{7}{24} \nu^{3}+\cdots, \\
& h \sim e^{t}\left(1-\frac{1}{2} \nu-\frac{1}{24} \nu^{2}+\cdots\right)=e^{t}-\frac{1}{2}-\sum_{k \geqslant 1} \gamma_{k} \nu^{k}
\end{aligned}
$$

при $\nu \rightarrow 0, \gamma_{1}=\frac{1}{24}$. Пусть $s(t)=\ln L(h)-t h+e^{t}-\frac{1}{2} t-\ln \sqrt{2 \pi}$, где $h=h(t)$ - решение уравнения (1.5). Имеем по (5.12) и (3.15) $s^{\prime}(t)=$ $-h+e^{t}-\frac{1}{2} \sim \sum_{k \geqslant 1} \gamma_{k} \nu^{k}, \nu \rightarrow 0$. Поскольку $s(\infty)=0$, отсюда следует $s(t) \sim-\int_{t}^{\infty} \sum_{k \geqslant 1} \gamma_{k} \nu^{k} d t=\sum_{k \geqslant 1} \gamma_{k} \nu^{k} / k, \nu \rightarrow 0$, или

$$
\ln L(h)-t h \sim \ln \sqrt{2 \pi}+\frac{1}{2} t-e^{t}+\sum_{k \geqslant 1} \frac{\gamma_{k}}{k} \nu^{k}, \quad \nu \rightarrow 0 .
$$

Далее, в соответствии с $(5.10), \sigma^{2}(h) \sim 1 / h-1 /\left(2 h^{2}\right)+\sum_{k \geqslant 1} B_{k} h^{-2 k-1}$, $h \rightarrow \infty$. Используя (5.11), отсюда заключаем

$$
\begin{aligned}
& \frac{1}{\sigma(h)} \sim \frac{1}{\sqrt{\nu}}\left(1+\frac{5}{48} \nu^{2}+\cdots\right), \\
& \frac{1}{h \sigma(h)} \sim \sqrt{\nu}\left(1+\frac{1}{2} \nu+\frac{19}{48} \nu^{2} \ldots\right), \quad \nu \rightarrow 0 .
\end{aligned}
$$

Рассуждая аналогично, покажем, что $a_{1}(h)=\frac{1}{24} \nu(1+O(\nu)), \nu \rightarrow 0$ (см. замечание 2). Нужные нам соотношения следуют из (5.10)-(5.14), теоремы $2 \mathrm{~B}$ и следствия $2 \mathrm{~B}$. 
$\mathrm{K}$ п р и м е р у С. Соответствующие результаты можно получить как прямое следствие теоремы $2 \mathrm{C}$, если вместо случайной величины $X_{1}$ рассмотреть $-X_{1}$ (а вместо $\left.p(x)-p(-x)\right)$. Заметим также, что в рассматриваемой ситуации $\lambda_{m+2}(h)=(-1)^{m}(m+1) ! \rho^{-m / 2}$ (см. замечание 2 ).

\section{СПИСОК ЛИТЕРАТУРЫ}

1. Боровков A.A., Рогозин Б. А. О центральной предельной теореме в многомерном случае. - Теория вероятн. и ее примен., 1965 , т. 10, в. 1, с. 61-69.

2. Daniels $H$. E. Saddlepoint approximations in statistics. - Ann. Math. Statist., 1954, v. 25 , № 4 , p. $631-650$.

3. Петров В. В. О вероятностях больших уклонений сумм независимых случайных величин. - Теория вероятн. и ее примен., 1965 , т. 10, в. 2 , с. 310-322.

4. Нагаев А. В. Большие уклонения для одного класса распределений. - Предельные теоремы теории вероятностей. Ташкент: АН УЗССР, 1963, с. 56-68.

5. Крамер Г. Об одной новой предельной теореме теории вероятностей. - Успехи матем. наук, 1944, № 10, с. 166-178.

6. Bahadur R. R., Ranga Rao R. On deviations of the sample mean. - Ann. Math. Statist., 1960, v. 31, № 4, p. 1015-1027.

7. Höglund T. A unified formulation of the central limit theorem for small and large deviations from the mean. - Z. Wahrscheinlichkeitstheor. verw. Geb., 1979, v. 49, p. $105-117$.

8. Феллер В. Введение в теорию вероятностей и ее приложения. Т. 1. М.: Мир, 1984, $527 \mathrm{c}$.

9. Петров В. В. Суммы независимых случайных величин. М.: Наука, 1972, 416 с.

10. Статулявичус $B$. A. Предельные теоремы для плотностей и асимптотические разложения для распределений сумм независимых случайных величин. - Теория вероятн. и ее примен., 1965 , т. 10 , в. 4, с. 645-659.

11. Розовский Л. В. Оценка интеграла от модуля характеристической функции обобшенного пуассоновского распределения по интервалу малой длины. - Зап. научн. семин. ПОМИ, 1999, т. 260, с. 240-249.

12. Хастингс Н., Пикок Дж. Справочник по статистическим распределениям. М.: Статистика, $1980,95 \mathrm{c}$.

13. Фихтенгольи Г. М. Курс дифференциального и интегрального исчисления. Т. 2. М.: Физматгиз, 1962,808 c.

14. Нагаев $A$. В. Большие уклонения для одного класса положительных случайных величин. - Изв. АН УзССР, 1963, т. 1, с. 18-20.

15. Нагаев A.B., Ходжабагян C.C. Предельные теоремы, учитывающие большие уклонения, для сумм положительных случайных величин. - Литов. матем. сб., 1974, т. 14 , в. 1, c. $149-163$.

16. Нагаев $A$. В. Большие уклонения для сумм решетчатых случайных величин при выполнении условия Крамера. - Дискретн. матем., 1998, т. 10, № 3, p. 115-130.

Поступила в редакцию

20.XII. 2000 